\title{
Finite Rotations
}

\author{
Oscar Bolina* \\ Department of Mathematics \\ University of California, Davis \\ Davis, CA 95616-8633 USA
}

\author{
J. A. da Silva Neto ${ }^{\dagger}$ \\ Instituto de Física \\ Universidade de São Paulo \\ Caixa Postal 66318 \\ 05315-970 São Paulo, Brasil
}

\begin{abstract}
We present an elementary discussion of two basic properties of angular displacements, namely, the anticommutation of finite rotations, and the commutation of infinitesimal rotations, and show how commutation is achieved as the angular displacements get smaller and smaller.
\end{abstract}

Key words: Finite Rotations, Commutivity

PACS numbers: $01.55,46.01 \mathrm{~B}$

\section{Introduction}

Even though finite rotations can be represented by a magnitude (equal to the angle of rotation) and a direction (that of the axis of rotation), they do not act like vectors. In particular, finite rotations do not commute: The summation of a number of finite rotations, not about the same axis, is dependent on the order of addition.

The anticommutivity property of finite rotations is made clear in introductory texts by showing that two successive finite rotations, when effected in different order, produce different final results [1].

However, when rotations are small - indeed, infinitesimal -, the combination of two or more individual rotations is unique, regardless of the order they are brought about (This fact allows for the

*Supported by FAPESP under grant 97/14430-2. E-mail: bolina@math.ucdavis.edu

${ }^{\dagger}$ Supported by FAPESP under grant 97/01003-9. E-mail: jantonio@fma.if.usp.br 
definition of angular velocity as the time-derivative of an angular coordinate [2, p.675]).

Here we show how the order rotations are carried out becomes irrelevant - that is, rotations become commutative - as the angles of rotation diminish.

In Fig. 1 we have represented two successive rotations of a rigid body. The first rotation is around the axis $O Z$ through an angle $\phi$, which takes $O A$ into $O B$. For simplicity, we take the plane $O A B$ to be the horizontal $X Y$ plane. The second rotation is around the axis $O X$ through an angle $\theta$, which takes $O B$ into $O C$.

Since the angle between the axes $O B$ and the axis of rotation $O X$ is not $90^{\circ}$, the plane $O B C$ cuts the plane $X Y$ at an angle. Let this angle be $\beta$, represented in Fig. 1 as the angle formed by the sides $P Q$ and $Q R$ of the triangle $P Q R$.

After these two rotations, the initial point $A$ is brought to the final position $C$. This same final result can be accomplished by just one rotation through an angle $(A, C)$ around an axis perpendicular to both $O B$ and $O C$.

To obtain a relation between the angles $\phi, \theta, \beta$ and $(A, C)$, we have drawn in Fig. 2 four triangles, derived from Fig. 1, which are relevant to our analysis.

From the two right triangles $O Q P$ and $O Q R$, we have the relations

$$
\cos \phi=\frac{O Q}{O P}, \quad \sin \phi=\frac{P Q}{O P}
$$

and

$$
\cos \theta=\frac{O Q}{O R}, \quad \sin \theta=\frac{Q R}{O R}
$$

The law of cosines applied to the triangles $O P R$ and $P Q R$ yields

$$
P R^{2}=O P^{2}+O R^{2}-2(O P)(O Q) \cos (A, C)
$$

and

$$
P R^{2}=P Q^{2}+Q R^{2}-2(P Q)(Q R) \cos \beta
$$

Substituting for $P Q$ and $Q R$ their values given in (1.1,1.2), Eq. (1.4) becomes

$$
P R^{2}=O P^{2} \sin ^{2} \phi+O R^{2} \sin ^{2} \theta-2(O P)(O R) \sin \phi \sin \theta \cos \beta
$$

On equating expressions (1.3) and (1.5) for $P R$, using (1.1,1.2), we get

$$
\cos (A, C)=\cos \phi \cos \theta+\sin \phi \sin \theta \cos \beta
$$


Now we effect the rotations in the reverse order, taking the first rotation around the axis $O X$ through an angle $\theta$, followed by a rotation around the axis $O Z$ through an angle $\phi$. In this case, the point $A$ moves to the new final position $E$, instead of $C$, as indicated in the sketch accompanying Fig. 1. The same final result can again be accomplished by just one rotation, now through an angle $(A, E)$ around an axis perpendicular to both $O D$ and $O E$.

A moment's reflection shows that the relation between the angles now is analogous to (1.6), with no need to repeat the above procedure. The cosine of the angle $(A, E)$ is given by $\cos (A, E)=$ $\cos \theta \cos \phi+\sin \theta \sin \phi \cos \beta^{\prime}$, with the difference that $\beta^{\prime}$ is the angle the plane $A O D$ makes with the horizontal plane DOE. Thus we have

$$
\cos (A, C)-\cos (A, E)=\sin \phi \sin \theta\left(\cos \beta-\cos \beta^{\prime}\right)
$$

If we set $\beta^{\prime}=\beta+\Delta \beta$, and expand $\cos \beta^{\prime}$ in (1.7) we get

$$
\cos (A, C)-\cos (A, E)=\sin \phi \sin \theta[\cos \beta(1-\cos \Delta \beta)+\sin \beta \sin \Delta \beta]
$$

To see how commutivity is obtained when the angles involved are small, we use that, for $x \ll 1$, $\sin x \approx x, \cos x \approx 1$. Eq. (1.8) becomes

$$
\cos (A, C)-\cos (A, E) \approx \phi \theta \Delta \beta \sin \beta
$$

This means that the difference between the two final positions vanishes more rapidly than either of the single rotations.

Remark: It is not necessary to assume that $\Delta \beta$ is small. Our result holds whether it is small or not, since in (1.7) we could have simplified our analysis by using that $\left|\cos \beta-\cos \beta^{\prime}\right| \leq 2$, and getting the same conclusion above.

\section{References}

[1] J. C. Slater, N. H. Frank, Mechanics, McGraw-Hill Book Company, N.Y. 1947, p.102-103

[2] A. P. French, Newtonian Mechanics, W. W. Norton Company, N.Y. 1973 

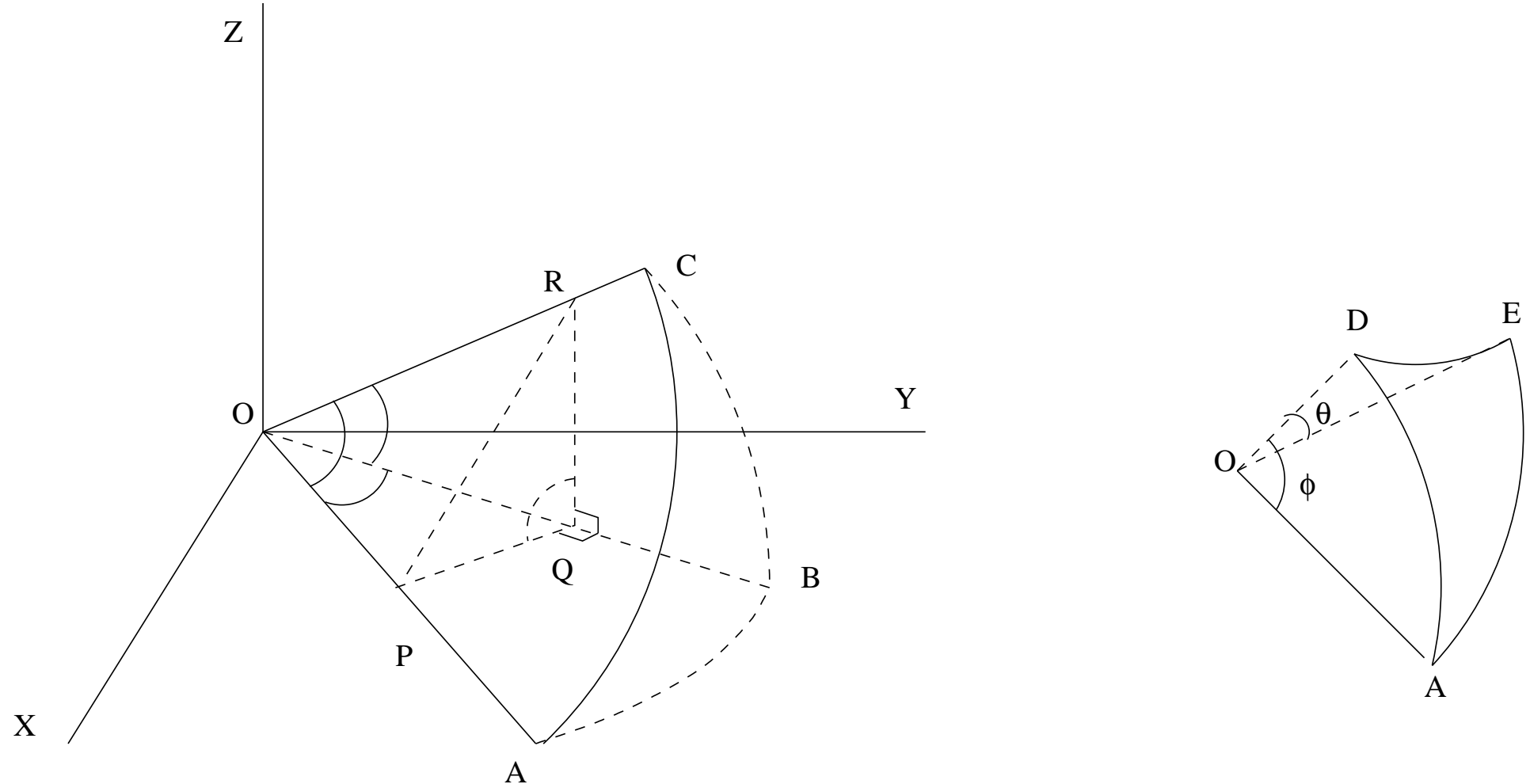

Figure 1: Two successive finite rotations

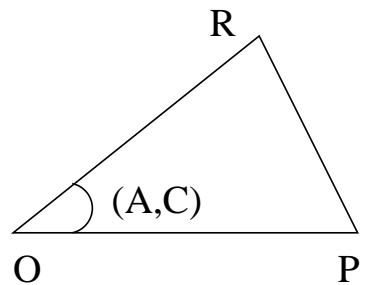

(1)

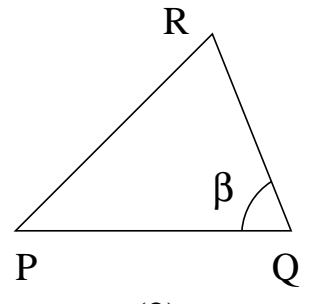

(2)

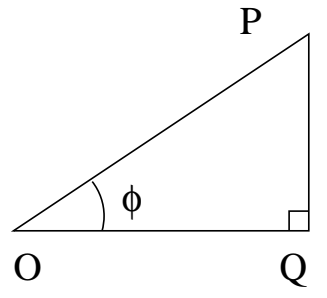

(3)

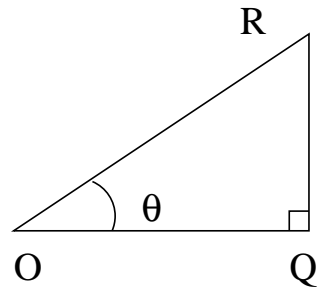

(4)

Figure 2: The Four Triangles 